\title{
Erratum: Hückel versus Möbius aromaticity: The particle in a cylinder versus a Möbius strip [Phys. Rev. A 82, 062118 (2010)]
}

\author{
Evangelos Miliordos
}

(Received 29 June 2016; published 15 July 2016)

DOI: 10.1103/PhysRevA.94.019904

In the titled article the single particle constrained in a Möbius strip was studied from the quantum-mechanical viewpoint. Unfortunately, the transformation of the Hamiltonian operator in Cartesian coordinates $(x, y$, and $z)$ to the internal coordinates $(s, \phi)$ is incorrect. C. C. Bastos, A. C. Pavão, E. S. G. Leandro have recently published the quantum mechanical problem of a particle in generalized cylinders and referred to this issue [1]. The proof provided in the Appendix of the original article is based on the expression of $s, \phi$ in terms of $x, y$, and $z$. Instead, the correct Laplacian is given by the Laplace-Beltrami operator,

$$
\nabla^{2}=|g|^{-1 / 2} \sum_{a, b=1}^{2} \frac{\partial}{\partial \sigma_{a}}|g|^{1 / 2} g_{a b} \frac{\partial}{\partial \sigma_{b}},
$$

where $\sigma_{1}=s, \sigma_{2}=\phi$, and $|g|$ is the determinant of the $g$ matrix given by

$$
(d x)^{2}+(d y)^{2}+(d z)^{2}=\sum_{a, b=1}^{2} g_{a b} d \sigma_{a} d \sigma_{b} .
$$

Next, we use that $d w=(\partial w / \partial s) d s+(\partial w / \partial \phi) d \phi$, where $w=x, y, z$. Exploiting Eq. (4) of the original article we come up with $(d x)^{2}+(d y)^{2}+(d z)^{2}=(d s)^{2}+\tau^{2}(d \phi)^{2}$, where

$$
\tau^{2}=[R+s \cos (\phi / 2)]^{2}+s^{2} / 4=r^{2}+s^{2} / 4 .
$$

Finally going back to the above Eq. (E1) we obtain

$$
\nabla^{2}=\frac{1}{\tau} \frac{\partial}{\partial s} \tau \frac{\partial}{\partial s}+\frac{1}{\tau} \frac{\partial}{\partial \phi} \frac{1}{\tau} \frac{\partial}{\partial \phi} .
$$

Consequently, the variable $r$ of Eq. (5) of the original article must be replaced by $\tau$, which goes to $r$, at first approximation, and finally to $R$ when $r \sim R \gg L>|s|$. Therefore the conclusions drawn in the original article are not affected. However, Eqs. (17)-(21) must be changed as follows:

$$
\begin{gathered}
a_{p, q}=\frac{1}{\delta^{2}}+\frac{4 r_{p, q} \cos \left(\phi_{q} / 2\right)+s_{p}}{8 \tau_{p, q}^{2} \delta}, \\
b_{p, q}=\frac{1}{\delta^{2}}-\frac{4 r_{p, q} \cos \left(\phi_{q} / 2\right)+s_{p}}{8 \tau_{p, q}^{2} \delta}, \\
c_{p, q}=\frac{1}{\tau_{p, q}^{2} \epsilon^{2}}+\frac{r_{p, q} s_{p} \sin \left(\phi_{q} / 2\right)}{4 \tau_{p, q}^{4} \epsilon}, \\
d_{p, q}=\frac{1}{\tau_{p, q}^{2} \epsilon^{2}}-\frac{r_{p, q} s_{p} \sin \left(\phi_{q} / 2\right)}{4 \tau_{p, q}^{4} \epsilon} \\
e_{p, q}=-2\left[\frac{1}{\delta^{2}}+\frac{1}{\tau_{p, q}^{2} \epsilon^{2}}\right] .
\end{gathered}
$$

Finally, the numerical results of Table I change slightly as shown in the new Table I. 
TABLE I. Energy levels $E$ (a.u.) of an electron confined to a Möbius strip with $R=5.67$ and $L=3.78$ a.u. The approximate energies $E_{n, m}$ (a.u.) as calculated from Eq. (8) are also given.

\begin{tabular}{|c|c|c|c|c|c|}
\hline State(s) & $t$ & & $E_{n, m}$ & $n$ & $m$ \\
\hline 1 & 0.345411 & & 0.345371 & 1 & 0 \\
\hline 2,3 & 0.361414 & 0.361335 & 0.360924 & 1 & \pm 1 \\
\hline 4,5 & 0.408739 & 0.408739 & 0.407582 & 1 & \pm 2 \\
\hline 6,7 & 0.487445 & 0.487445 & 0.485345 & 1 & \pm 3 \\
\hline 8,9 & 0.597063 & 0.597063 & 0.594213 & 1 & \pm 4 \\
\hline 10,11 & 0.737028 & 0.737028 & 0.734187 & 1 & \pm 5 \\
\hline 12,13 & 0.906640 & 0.906640 & 0.905266 & 1 & \pm 6 \\
\hline 14,15 & 1.105089 & 1.105089 & 1.107450 & 1 & \pm 7 \\
\hline 16,17 & 1.331494 & 1.331494 & 1.340740 & 1 & \pm 8 \\
\hline 18,19 & 1.384986 & 1.384986 & 1.385373 & 2 & $\pm 1 / 2$ \\
\hline 20,21 & 1.418194 & 1.418194 & 1.416478 & 2 & $\pm 3 / 2$ \\
\hline 22,23 & 1.482914 & 1.482914 & 1.478689 & 2 & $\pm 5 / 2$ \\
\hline 24,25 & 1.580113 & 1.580113 & 1.572005 & 2 & $\pm 7 / 2$ \\
\hline 26,27 & 1.584945 & 1.584945 & 1.605134 & 1 & \pm 9 \\
\hline 28,29 & 1.709906 & 1.709906 & 1.696426 & 2 & $\pm 9 / 2$ \\
\hline 30,31 & 1.864554 & 1.864554 & 1.851952 & 2 & $\pm 11 / 2$ \\
\hline 32,33 & 1.872426 & 1.872426 & 1.900634 & 1 & \pm 10 \\
\hline 34,35 & 2.067780 & 2.067780 & 2.038584 & 2 & $\pm 13 / 2$ \\
\hline
\end{tabular}

I would like to express my gratitude to Dr. G. Schenter (Pacific Northwest National Laboratory, Richland, WA) for bringing to my attention the above issue. I am also thankful to C. C. Bastos, A. C. Pavão, and E. S. G. Leandro for their careful review of the present erratum.

[1] C. C. Bastos, A. C. Pavão, and E. S. G. Leandro, J. Math. Chem. 1 (2016), doi:10.1007/s10910-016-0652-5. 